\title{
Second and subsequent tumours among 1927 retinoblastoma patients diagnosed in Britain 1951-2004
}

\author{
A MacCarthy ${ }^{\star 1}$, A M Bayne ${ }^{1}$, P A Brownbill ${ }^{1,5}, \mathrm{~K} \mathrm{~J} \mathrm{Bunch}^{1}$, N L Diggens ${ }^{1}$, G J Draper ${ }^{1}$, M M Hawkins ${ }^{2}$, \\ H C Jenkinson ${ }^{3}$, J E Kingston ${ }^{4}$, C A Stiller ${ }^{1}$, T J Vincent ${ }^{1}$ and M F G Murphy ${ }^{1}$ \\ ${ }^{1}$ Childhood Cancer Research Group, New Richards Building, University of Oxford, Old Road Campus, Headington, Oxford OX3 \\ 7LG, UK; ${ }^{2}$ Centre for Childhood Cancer Survivor Studies, School of Health and Population Sciences, University of Birmingham, \\ Edgbaston, Birmingham B15 2TT, UK; ${ }^{3}$ Retinoblastoma Service, Birmingham Children's Hospital Foundation NHS Trust, \\ Birmingham B4 6NH, UK and ${ }^{4}$ Retinoblastoma Service, Barts Health NHS Trust, London E1 1BB, UK
}

Background: Retinoblastoma is an eye tumour of childhood that occurs in heritable and non-heritable forms. In the heritable form, there is a predisposition to the development of non-ocular subsequent primary tumours (SPTs).

Methods: This study included 1927 retinoblastoma patients diagnosed in Britain from 1951 to 2004 . Ascertainment was through the (UK) National Registry of Childhood Tumours; cases were followed-up for the occurrence of SPTs. Standardised incidence ratios (SIRs) were calculated.

Results: We identified 169 SPTs in 152 patients. The SIR analysis included 145 SPTs with cancer registrations from the years 1971 to 2009. These tumours occurred in 132 patients: 112 of the 781 heritable and 20 of the 1075 (presumed) non-heritable cases under surveillance at the start of this period developed at least one registered SPT. The SIRs for all tumours combined were 13.7 (95\% confidence interval 11.3-16.5) in heritable cases and 1.5 (0.9-2.3) in non-heritable cases. The main types of SPT in the heritable cases were leiomyosarcoma, (31 cases; SIR 1018.7 (692.2-1446.0)), osteosarcoma (26 cases; SIR 444.6 (290.4-651.4)), and skin melanoma (12 cases; SIR 18.6 (9.6-32.4)).

Conclusion: The risk of SPTs in heritable retinoblastoma is extremely high. This has important implications for the clinical follow-up and counselling of survivors and their families.

Retinoblastoma (Online Mendelian Inheritance in Man, OMIM, 2012) occurs in two forms: heritable, in $\sim 45 \%$ of cases, and non-heritable. The heritable form of the disease involves a germline mutation in one allele of the $R B 1$ gene followed by an acquired mutation in the second allele. The initial mutation may arise de novo or be inherited from a parent. The non-heritable form of the disease involves two post-conception mutational events in one retinal cell. It is well established that there is an increased risk of subsequent primary tumours (SPTs) following retinoblastoma in heritable cases and that this increased risk extends into adult life and involves many different tumour types.
We examine the occurrence of SPTs in two large cohorts of retinoblastoma cases, consisting respectively of heritable and (presumed) non-heritable cases. Each of these cohorts is unbiased with respect to case selection and follow-up.

\section{MATERIALS AND METHODS}

The study group, which is described in more detail in a previous paper (MacCarthy et al, 2009), comprised 1927 cases of

*Correspondence: A MacCarthy; E-mail: angela.maccarthy@ccrg.ox.ac.uk

${ }^{5}$ Previously a member of Childhood Cancer Research Group staff

Received 20 December 2012; revised 5 April 2013; accepted 17 April 2013; published online 14 May 2013

(c) 2013 Cancer Research UK. All rights reserved 0007-0920/13 
retinoblastoma diagnosed in Great Britain from 1951 to 2004 ascertained through the National Registry of Childhood Tumours (NRCT) (Stiller, 2007). Nationally, 1716 cases of retinoblastoma were registered from 1962 to 2004. A further unselected 211 cases diagnosed 1951-1961 from certain cancer registries and treatment centres were included. The study comprises two individually unbiased cohorts: 806 'heritable' cases and 1121 (presumed) 'non-heritable' cases: bilateral cases and those with a family history of retinoblastoma are classified as 'heritable'; the remainder (1101 unilateral cases and 20 of unknown laterality) are classified here as 'non-heritable'. Cases classified as 'non-heritable' may in fact include a proportion of heritable cases: $\sim 15-20 \%$ of unilateral cases are thought to be heritable, though only some will be recognised as such. (We believe that the word 'heritable' is to be preferred to the frequently used 'hereditary', as for many of these cases there is no family history.)

Subsequent primary tumours were identified through various sources, mainly notifications of cancer registrations received from the National Health Service Information Centre, Medical Research Information Service (England and Wales) and the General Register Office (Scotland); pathology was verified by obtaining a copy of the diagnostic report or through confirmation of the tumour by the hospital consultant. Subsequent primary tumours occurring in study cases were included in the analysis only if they had cancer registrations, because as explained below, cancer registry data were used in calculating the numbers of SPT cases expected. The analysis was confined to the period 1971-2009-the period for which surveillance through cancer registration was, as far as possible, complete. For each case, the actual period of surveillance (or follow-up) is the interval between the entry date and the exit date, these dates being defined as follows: entry date is the later of diagnosis date and 1 January 1971. Exit date is the earliest of 31 December 2009, date lost to follow-up, date of death, date of the first SPT of the type currently being analysed. The expected numbers of tumours (in total or for specified diagnostic groups) were calculated by multiplying the person-years at risk (subdivided according to age-group and calendar period) for each case during the period of surveillance by the age-sex-period rates from the national cancer registration data for England and Wales 1971-2006, the rates for 2007-9 being assumed to be the same as for 2006, and the rates for Scotland being assumed to be the same as for England and Wales.

Brain and central nervous system tumours were included whether or not specified as malignant; this follows the policy of cancer registries. Skin carcinomas, primitive neuroectodermal tumours in the naso-ethmoid region and 'trilateral retinoblastoma' were excluded (MacCarthy et al, 2009).

The diagnostic groupings of SPTs in the analyses described below were chosen so that the rates for specified sites and histological types could be compared with those for other major studies. In the tables and analyses below, SPTs are categorised sometimes by site and sometimes by type; thus, some are included in more than one group. In addition, sites were grouped as 'inside head and neck' (including tumours in the thyroid gland) or 'outside head and neck', as a way of comparing anatomical regions with higher and lower potential exposure to radiotherapy that may have been used in treatment.

The numbers of SPTs among the retinoblastoma patients were compared with those expected in the general population using standardised incidence ratios (SIRs).

The SIR is defined here as the ratio of the observed number of SPTs to the number expected from population incidence rates based on national cancer registration data for England and Wales for the period 1971-2006. (Note that, in contrast to the frequent convention, the ratio is not multiplied by 100). Standardised incidence ratios and 95\% confidence intervals (CI) were calculated using STATA version 11 (StataCorp., 2009). Tests of the hypothesis that an SIR is equal to one are based on two-sided $P$-values. Tests of equality between two SIRs were based on calculations using the (binomial) conditional distribution for comparing two Poisson variables (Breslow and Day, 1987).

The absolute excess risk (AER) corresponding to each SIR was also calculated. This is an estimate of the increased risk in the study group as compared with the general population and gives a direct measure of the impact of a factor on risk. The units in which such risks are expressed vary. Here we use AERs per 100000 personyears; these figures are directly comparable with the rates in which cancer registration data are usually expressed.

Some heritable cases developed more than one SPT. The SIR for all tumour types combined was calculated using only the first SPT. The SIRs for individual types of SPT were calculated using the first SPT of that type; for some cases this will have been the second or third subsequent tumour. The rationale for including only the first SPT of a particular type in these analyses is that our objective is to analyse the risk to a retinoblastoma patient of at least one such event occurring. This will lead to some underestimation of the SIR, as the population data used in calculating the expected numbers will include registrations for both the initial tumour and also any subsequent tumours occurring in the same patient. However, the latter can be expected to account for only a small proportion of the total cancer registrations $(\sim 5 \%$ at all ages in Scotland (Sasieni et al, 2011), Denmark (Storm et al, 1985), Connecticut (Boice et al, 1986)), and the resulting underestimation of SIRs will be small.

A limitation of the present study is that we do not have data on either radiotherapy or chemotherapy; the consequences of this are considered further in the Discussion.

\section{RESULTS}

In Table 1, we summarise the characteristics of the cases in terms of sex, year of diagnosis and laterality. We identified and validated 169 SPTs occurring in 152 patients (Table 2). Of these 169 tumours, 3 occurred before 1971, 18 did not have a cancer registration in Britain and 3 occurred after 2009. There were thus

\begin{tabular}{|c|c|c|}
\hline & $\begin{array}{c}\text { No. of heritable } \\
\text { cases }(\%)\end{array}$ & $\begin{array}{c}\text { No. of non-heritable } \\
\text { cases (\%) }\end{array}$ \\
\hline Total & 806 (100) & $1121(100)$ \\
\hline Male & $446(55.3)$ & $541(48.3)$ \\
\hline Female & $360(44.7)$ & $580(51.7)$ \\
\hline \multicolumn{3}{|c|}{ Age at diagnosis (years) } \\
\hline 0 & 509 (63.2) & $226(20.2)$ \\
\hline 1 & 191 (23.7) & $293(26.1)$ \\
\hline $2+$ & $106(13.2)$ & $602(53.7)$ \\
\hline \multicolumn{3}{|c|}{ Diagnosed } \\
\hline $1951-1961$ & $104(12.9)$ & $107(9.6)$ \\
\hline 1962-2004 & 702 (87.1) & $1014(90.4)$ \\
\hline \multicolumn{3}{|l|}{ Laterality } \\
\hline Bilateral & $728(90.3)$ & $0(0.0)$ \\
\hline Unilateral & $77(9.6)$ & 1101 (98.2) \\
\hline Unknown & $1(0.1)$ & $20(1.8)$ \\
\hline \multicolumn{3}{|c|}{$\begin{array}{l}\text { Heritable cases make up } 42 \% \text { of the total. The proportion is higher among children } \\
\text { diagnosed before 1962; because for that period, the study includes a higher proportion of } \\
\text { cases from specialist centres and such cases were more likely to be bilateral/heritable. The } \\
728 \text { bilateral cases are all classified as heritable. There are also } 77 \text { unilateral cases and one of } \\
\text { unknown laterality that had a family history and is therefore also classified as heritable. }\end{array}$} \\
\hline
\end{tabular}




Table 2. Validated subsequent primary tumours
\begin{tabular}{|l|c|c|c|}
\hline $\begin{array}{l}\text { Diagnostic } \\
\text { group }\end{array}$ & $\begin{array}{c}\text { Number of } \\
\text { tumours in } \\
\text { heritable cases }\end{array}$ & $\begin{array}{c}\text { Number of } \\
\text { tumours in non- } \\
\text { heritable cases }\end{array}$ & Total \\
\hline All tumours & $146(112)$ & $23(20)$ & $169(132)$ \\
Bone tumours & $34(27)$ & $4(2)$ & $38(29)$ \\
$\quad$ Osteosarcoma & $33(26)$ & $3(1)$ & $36(27)$ \\
Soft-tissue & $51(46)$ & $1(1)$ & $52(47)$ \\
sarcomas & & & \\
$\quad$ Leiomyosarcoma & $33(31)$ & $1(1)$ & $34(32)$ \\
Melanoma of skin & $14(12)$ & $2(2)$ & $16(14)$ \\
Leukaemia & $5(3)$ & $1(1)$ & $6(4)$ \\
Brain/central & $15(12)$ & $4(3)$ & $19(15)$ \\
nervous system & & $3(2)$ & $12(10)$ \\
Meningioma & $9(8)$ & $1(1)$ & $9(9)$ \\
Bladder & $8(8)$ & 0 & $6(3)$ \\
Trachea, bronchus & $6(3)$ & $2(2)$ & $11(10)$ \\
and lung & $9(8)$ & 0 & $7(7)$ \\
Female breast & $7(7)$ & $2(2)$ & $3(3)$ \\
Uterus & $1(1)$ & &
\end{tabular} Testis

${ }^{a}$ One of the subsequent primary tumours in the non-heritable group was a leiomyosarcoma of bone. In this and subsequent tables, this tumour is included as one of the 'Soft Tissue Sarcomas' and also in the total 'Bone tumours'.

These 169 subsequent primary tumours occurred in 152 retinoblastoma cases. 138 patients had one subsequent primary tumour, 11 had two and 3 had three. The diagnostic groups are not all included here, and those that are included are not mutually exclusive, for example, a bladder sarcoma would be counted in the groups 'Soft-tissue sarcomas' and 'Bladder'. Numbers in brackets are those tumours included in the SIR analyses reported in Table 4; all of these were registered in the period 1971-2009.Diagnostic groups are defined using ICD8 site codes, MOTNAC (Manual of Tumour Nomenclature and Classification) type code for the period 1971-1978. ICD 9 and ICD-O were used for 1979-1994 and ICD 10 and ICD-O-2 for 1995-2009.

145 SPTs (in 132 patients) that had cancer registrations between 1971 and 2009 among the 1856 cases under surveillance for varying periods during these years; analyses of SIRs and Tables 3, 4,5 and 6 , are based on this group.

Table 3 shows these 1856 cases subdivided by sex and heritability together with person-years of surveillance for each age interval. Table 4 shows detailed results for groups of SPTs among heritable cases and numbers of such tumours among non-heritable cases.

The high incidence of SPTs is almost entirely confined to the heritable cases. In this group, the SIR for all tumour types combined was 13.7 (95\% confidence interval 11.3-16.5) based on 112 second tumours. The effect is highly significant $(P<0.001)$ for each sex. The SIR for males, 17.4 is significantly greater than that for females, $10.9(P<0.05)$; this sex difference could in theory be attributable to confounding, for example, by diagnostic group, age or calendar year, though the effect is seen in many diagnostic subgroups, and in four out of five of the age groups shown in Table 5. The overall AER is 579.0 (95\% CI 468.7-706.0) per 100000 person-years. For the heritable cases, all categories included in Table 4 (which were chosen on the basis of findings from previous studies or likely radiation risks) show a raised risk, and all except those for leukaemia and testis are statistically significant. The most common type of SPT in the heritable cases was soft-tissue sarcoma (46 cases), including leiomyosarcoma (31 cases), which was the tumour type with the highest SIR (around 1000) for both males and females. There were also 27 bone tumours, of which 26 were osteosarcomas, the SIRs for the latter being the second highest for both males and females. There were 12 cases of melanoma and 12 $\mathrm{CNS} /$ Brain tumours, of which 8 were meningiomas. Only three leukaemias were included in the analyses of SIRs for the heritable cases; two others were ascertained (Table 2) but were not eligible for these analyses. The one leukaemia in a non-heritable case occurred in a patient with Fanconi anaemia. Particularly, high SIRs were also found for tumours of the bladder and uterus, with high AERs for uterus and female breast (Table 4). In Table 5, we examine further the results for the main diagnostic groups, giving SIRs and AERs by age and sex for the heritable cases only. For all diagnoses taken together, the SIRs show some tendency to decrease with age, whereas the AERs increase. For osteosarcoma, the SIRs at younger ages are particularly high. But perhaps the most remarkable finding in this table is the very large increase in AER with increasing age for leiomyosarcoma. The sex difference, higher SIRs for males than females, is also seen for osteosarcoma and melanoma and within nearly all of the individual age groups for these diagnoses.

The above results for the heritable cases may be contrasted with those for the non-heritable. In the latter, we found a small nonsignificant increase in the 'All tumours' group: SIR 1.5 (0.9-2.3) based on 20 second tumours; there were also usually small, usually non-significant increases in some of the individual groups.

We do not at present have adequate data to allow an analysis of the effects of treatment on the incidence of SPTs. It is, however, possible to attempt some assessment of the effects of radiotherapy by comparing SIRs within and outside the region of potential exposure to radiation. In Table 6, we compare SIRs for tumours within the head and neck with those for tumours outside this region, separately for heritable and non-heritable cases. Both within and outside the head and neck region, the SIRs for the heritable cases are 8-10 times those for the non-heritable cases. Similarly, both for the heritable and non-heritable cases, the head and neck SIRs are 2-3 times than those outside this region. The SIR for head and neck region tumours for heritable cases is about 25 times that for those outside this region in the non-heritable cases. This analysis suggests that there is a possible multiplicative effect of having an $R B 1$ gene mutation and being irradiated. This interpretation is subject to many caveats; in particular, we do not know whether all the cases with SPTs in the head and neck region were irradiated or whether heritable cases had more radiation. There are many other ways in which the two regions are not comparable, most obviously in the types of tumours occurring.

Some previous papers have analysed SIRs/standardised mortality ratios (SMRs) in terms of tumour sites. Table 7 shows, for the 169 validated SPTs included in Table 2, numbers of cases by site and histological type.

\section{DISCUSSION}

Major strengths of our study include its size and unbiased follow-up, independent of events subsequent to retinoblastoma such as attendance at hospital. However, there may be heritable cases that are misclassified as non-heritable. This may, in part, account for the raised risks seen in the non-heritable group, though the possible effect of treatment should also be considered.

Our findings are consistent with those from earlier studies in that the incidence of second (and subsequent) tumours in retinoblastoma cases is very much higher than would be expected from rates in the general population; this increased risk is almost entirely observed in the heritable group. The increased risk for osteosarcoma and softtissue sarcoma in the heritable group is well documented in earlier reports. Here, we also highlight a very high risk for leiomyosarcoma and examine the increase in the excess risk with increasing age.

We may compare the results for heritable cases from the present study with those from other studies based on unselected groups of heritable cases. Rates for groups described as 'non-heritable' are relatively low, and are unlikely to be comparable between series because of the varying proportions of unrecognised heritable cases that are included. 
Even for the heritable group, there are a number of factors that make it difficult to compare different series: some include pineoblastomas, others do not; treatment regimens vary; adequacy

Table 3. Numbers of cases under surveillance during varying periods between 1 January 1971 and 31 December 2009, and person-years of surveillance for the period 1971-2009

Numbers of cases under surveillance during varying periods between 1 January 1971 and 31 December 2009

Heritable cohort Non-heritable cohort

\begin{tabular}{|l|c|c|c|c|c|}
\hline Male & Female & Total & Male & Female & Total \\
\hline 432 & 349 & 781 & 519 & 556 & 1075 \\
\hline
\end{tabular}

Person-years of surveillance during specified age ranges Heritable cohort Non-heritable cohort

\begin{tabular}{|l|c|c|c|c|c|c|}
\hline $\begin{array}{l}\text { Age } \\
\text { (years) }\end{array}$ & Male & Female & Total & Male & Female & Total \\
\hline $0-14$ & 4261.2 & 3398.8 & 7660.0 & 4963.5 & 5266.9 & 10230.4 \\
\hline $15-24$ & 2450.4 & 2125.2 & 4575.6 & 3279.6 & 3366.7 & 6646.3 \\
\hline $25-34$ & 1706.6 & 1522.7 & 3229.3 & 2291.4 & 2300.5 & 4591.9 \\
\hline $35-44$ & 1027.8 & 854.6 & 1882.4 & 1312.5 & 1405.6 & 2718.1 \\
\hline $45-54$ & 280.3 & 263.7 & 544.0 & 521.8 & 483.1 & 1004.9 \\
\hline $55-64$ & 8.6 & 31.3 & 39.9 & 89.0 & 51.1 & 140.1 \\
\hline Total 0-64 & 9734.8 & 8196.3 & 17931.1 & 12457.8 & 12873.9 & 25331.7 \\
\hline
\end{tabular}

Table 4. Numbers of first subsequent primary tumours ${ }^{a}$ in each specified diagnostic group

Heritable cases

Non-heritable cases

\begin{tabular}{|c|c|c|c|c|c|c|c|c|c|c|c|c|}
\hline \multirow{4}{*}{$\begin{array}{l}\text { Diagnostic group } \\
\text { All tumours combined }\end{array}$} & \multicolumn{9}{|c|}{ Heritable cases } & \multicolumn{3}{|c|}{ Non-heritable cases } \\
\hline & \multicolumn{3}{|c|}{ Male } & \multicolumn{3}{|c|}{ Female } & \multicolumn{3}{|c|}{ Total } & Male & \multicolumn{2}{|c|}{ Female Total } \\
\hline & No. & SIR (95\% CI) & AER & No. & SIR $(95 \% \mathrm{Cl})$ & AER & No. & SIR $(95 \% \mathrm{Cl})$ & AER & \multicolumn{3}{|c|}{ No. of cases } \\
\hline & 61 & $17.4(13.3-22.4)$ & 590.6 & 51 & $10.9(8.1-14.3)$ & 565.2 & 112 & $13.7(11.3-16.5)$ & 579.0 & 13 & 7 & 20 \\
\hline All bone tumours ${ }^{b}$ & 21 & $262.6(162.6-401.4)$ & 210.4 & 6 & $114.2(41.9-248.5)$ & 70.9 & 27 & $203.7(134.3-296.4)$ & 146.6 & 2 & 0 & 2 \\
\hline Osteosarcoma & 20 & $563.9(344.4-870.8)$ & 200.6 & 6 & $260.8(95.7-567.6)$ & 71.3 & 26 & $444.6(290.4-651.4)$ & 141.5 & 1 & 0 & 1 \\
\hline All soft-tissue sarcomas ${ }^{\mathrm{b}}$ & 20 & $123.3(75.3-190.5)$ & 200.4 & 26 & $224.3(146.5-328.6)$ & 308.9 & 46 & $165.4(121.1-220.6)$ & 250.1 & 1 & 0 & 1 \\
\hline Leiomyosarcoma $^{\mathbf{b}}$ & 12 & $1061.0(548.2-1853.4)$ & 120.1 & 19 & 993.7 (598.3-1551.8) & 225.9 & 31 & $1018.7(692.2-1446.0)$ & 168.5 & 1 & 0 & 1 \\
\hline Rhabdomyosarcoma & 4 & $127.1(34.6-325.3)$ & 39.7 & 2 & $112.3(13.6-405.6)$ & 23.5 & 6 & $121.8(44.7-265.0)$ & 32.3 & 0 & 0 & 0 \\
\hline Fibrosarcoma & 0 & $0.0(0.0-89.0)$ & - & 2 & $68.1(8.2-246.0)$ & 23.4 & 2 & $31.7(3.8-114.6)$ & 10.5 & 0 & 0 & 0 \\
\hline Liposarcoma & 3 & $229.7(47.4-671.3)$ & 29.9 & 0 & $0.0(0.0-321.8)$ & - & 3 & $134.1(27.7-391.9)$ & 16.2 & 0 & 0 & 0 \\
\hline Other soft-tissue sarcomas & 1 & $13.1(0.3-73.2)$ & 9.2 & 3 & $72.2(14.9-211.2)$ & 35.1 & 4 & $34.0(9.3-87.1)$ & 21.0 & 0 & 0 & 0 \\
\hline Melanoma of skin & 7 & $26.6(10.7-54.7)$ & 67.3 & 5 & $13.1(4.2-30.5)$ & 55.0 & 12 & $18.6(9.6-32.4)$ & 61.7 & 0 & 2 & 2 \\
\hline CNS/Brain & 5 & $11.7(3.8-27.3)$ & 45.7 & 7 & $20.9(8.4-43.0)$ & 79.4 & 12 & $15.8(8.1-27.5)$ & 61.1 & 2 & 1 & 3 \\
\hline Meningioma & 3 & $118.8(24.5-347.2)$ & 29.7 & 5 & $100.9(32.8-235.4)$ & 59.0 & 8 & $106.9(46.2-210.7)$ & 43.1 & 1 & 1 & 2 \\
\hline Other CNS/Brain & 2 & $5.0(0.6-17.9)$ & 15.9 & 2 & $6.9(0.8-25.0)$ & 20.3 & 4 & $5.8(1.6-14.8)$ & 17.9 & 1 & 0 & 1 \\
\hline Leukaemia & 3 & $8.1(1.7-23.7)$ & 26.2 & 0 & $0.0(0.0-12.8)$ & - & 3 & $5.0(1.0-14.5)$ & 13.0 & 0 & 1 & 1 \\
\hline Bladder & 5 & $73.0(23.7-170.4)$ & 49.4 & 3 & $107.4(22.2-313.9)$ & 35.2 & 8 & $83.0(35.8-163.5)$ & 42.9 & 1 & 0 & 1 \\
\hline Trachea, bronchus, lung & 2 & $11.5(1.4-41.5)$ & 18.2 & 1 & $6.8(0.2-37.8)$ & 10.1 & 3 & $9.3(1.9-27.2)$ & 14.5 & 0 & 0 & 0 \\
\hline Female breast & - & - & - & 8 & $4.5(2.0-8.9)$ & 74.3 & 8 & $4.5(2.0-8.9)$ & 74.3 & - & 2 & 2 \\
\hline Uterus & - & - & - & 7 & $61.7(24.8-127.2)$ & 81.8 & 7 & $61.7(24.8-127.2)$ & 81.8 & - & 0 & 0 \\
\hline Testis & 1 & $1.8(0.0-10.0)$ & 4.4 & - & - & - & 1 & $1.8(0.0-10.0)$ & 4.4 & 2 & - & 2 \\
\hline $\begin{array}{l}\text { Abbreviations: AER = absolute } \\
\text { heritable cases. } \\
\text { a An SPT for any case is included } \\
\text { (see Materials and Methods). The } \\
\text { bone of the subsequent primary } \\
\text { and also in the total 'Bone tumo }\end{array}$ & 100 & $\begin{array}{l}\text { groups in this table a } \\
\text { the non-heritable gro }\end{array}$ & . & me & clusive, for example, a & 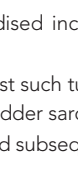 & D & Id occur in the grou & - & es & $\begin{array}{l}\text { cancer } \\
\text { mas' an }\end{array}$ & ation \\
\hline
\end{tabular}

and the length of follow-up differ. Also, comparisons of SIRs between studies must be interpreted with great caution: in particular, they are, in general, highly dependent on the ages to which cases are followed-up.

Previously, Draper et al (1986) presented data covering the whole of Great Britain for the diagnosis period 1962-77. Here we extend the period of national coverage to 2004 and include also a further group of retinoblastoma cases diagnosed 1951-1961. Fletcher et al (2004) studied 451 cases in Britain born before 1951 and followed from age 25 years. They concluded that survivors of heritable retinoblastoma had a high lifetime risk of developing epithelial tumours subsequent to retinoblastoma, even in the absence of high-dose radiotherapy. Comparisons between our current study and that by Fletcher et al are complicated by the fact that they report SMRs and the second tumours are differently classified.

A major series from the Netherlands, most recently reported by Marees et al (2008), included 668 cases of retinoblastoma diagnosed from 1945 to 2005 . For heritable cases, after a median follow-up of 21.9 years the risk of second malignancy was twenty times that found in the general Dutch population.

Kleinerman et al (2005) presented an analysis of 1601 retinoblastoma cases from two US medical centres diagnosed between 1914 and 1984. The authors later reported on the 69 soft-tissue sarcomas in cases among the 963 heritable cases in this cohort and found a statistically significant excess of soft-tissue sarcomas, particularly leiomyosarcoma, among the heritable cases.

Our SIR for 'all tumour types combined' following heritable retinoblastoma was significantly raised and broadly in line with SIRs and SMRs for heritable cases quoted in other large series 
Table 5. SIRs and AERs for age/sex groups. Major diagnostic categories, heritable cases only

\begin{tabular}{|c|c|c|c|c|c|c|c|c|}
\hline & \multicolumn{2}{|c|}{ All tumours combined } & \multicolumn{2}{|c|}{ Osteosarcoma } & \multicolumn{2}{|c|}{ Leiomyosarcoma } & \multicolumn{2}{|c|}{ Melanoma of skin } \\
\hline Age (years) & $\operatorname{SIR}(95 \% \mathrm{CI})$ & AER & SIR $(95 \% \mathrm{Cl})$ & AER & SIR $(95 \% \mathrm{CI})$ & AER & $\operatorname{SIR}(95 \% \mathrm{CI})$ & AER \\
\hline \multicolumn{9}{|l|}{ Male } \\
\hline $0-14$ & $25.5(14.3-42.1)$ & 338.2 & $832.1(415.4-1488.8)$ & 256.7 & 0.0 & - & $256.4(6.5-1428.6)$ & 23.2 \\
\hline $15-24$ & $27.9(15.6-46.0)$ & 590.2 & $462.2(199.5-910.6)$ & 322.8 & $1626.0(196.9-5873.7)$ & 79.4 & 0.0 & - \\
\hline $25-34$ & $13.0(6.2-23.8)$ & 540.8 & 0.0 & - & $307.7(7.8-1714.4)$ & 56.8 & $55.3(15.1-141.7)$ & 224.8 \\
\hline $35-44$ & $14.4(7.7-24.6)$ & 1177.0 & $699.3(17.7-3896.2)$ & 91.2 & $1522.8(558.9-3314.6)$ & 555.8 & $9.8(0.2-54.8)$ & 82.0 \\
\hline $45-54$ & $12.4(5.4-24.4)$ & 2623.9 & 0.0 & - & $1449.3(298.9-4235.4)$ & 949.2 & $19.1(0.5-106.5)$ & 285.1 \\
\hline \multicolumn{9}{|l|}{ Female } \\
\hline $0-14$ & $21.1(9.1-41.5)$ & 224.2 & $380.2(103.6-973.5)$ & 116.8 & 0.0 & - & 0.0 & - \\
\hline $15-24$ & $29.0(15.0-50.6)$ & 545.1 & $222.0(26.9-801.8)$ & 92.8 & $1234.6(149.5-4459.7)$ & 92.3 & $59.0(12.2-172.4)$ & 136.9 \\
\hline $25-34$ & $9.9(4.8-18.3)$ & 590.7 & 0.0 & - & $1176.5(320.6-3012.2)$ & 253.2 & $7.7(0.2-43.1)$ & 55.4 \\
\hline $35-44$ & $6.5(3.1-11.9)$ & 989.0 & 0.0 & - & 857.1 (314.6-1865.6) & 664.2 & $8.0(0.2-44.3)$ & 96.4 \\
\hline $45-54$ & $10.0(5.0-17.9)$ & 3754.3 & 0.0 & - & 1176.5 (473.0-2424.0) & 2307.7 & 0.0 & - \\
\hline
\end{tabular}

Table 6. SIRs for subsequent primary tumours within and outside head and neck region

\begin{tabular}{|l|c|c|c|c|c|c|}
\cline { 2 - 7 } \multicolumn{2}{|c|}{} & \multicolumn{3}{|c|}{ Within head and neck region } & \multicolumn{3}{c|}{ Outside head and neck region } \\
\hline & Person-years & No. of SPTs & SIR (95\% Cl) & Person-years & No. of SPTs & SIR (95\% CI) \\
\hline Heritable retinoblastoma & 18274.9 & 45 & $32.1(23.4-43.0)$ & 18114.5 & 70 & $10.0(7.8-12.7)$ \\
\hline Non-heritable retinoblastoma & 25367.2 & 6 & $2.9(1.1-6.3)$ & 25388.6 & 14 & $1.3(0.7-2.1)$ \\
\hline $\begin{array}{l}\text { Abbreviations: Cl= confidence interval; SIR = standardised incidence ratio (observed number/expected number); SPT = subsequent primary tumour. Some heritable cases had subsequent } \\
\text { primary tumours both within and outside the head and neck region. }\end{array}$ \\
\hline
\end{tabular}

(Fletcher et al, 2004; Kleinerman et al, 2005; Marees et al, 2008). Differences between these ratios reflect, in part, the underlying population risks of tumour development in different study populations. We found a significantly higher SIR in males than in females; Marees et al (2008) reported no significant difference.

Bone tumours were almost entirely osteosarcomas, a similar finding to that of previous studies (Kleinerman et al, 2005; Marees et al, 2008). In heritable cases, 10 osteosarcomas were classified 'in the head and neck' and 16 as 'outside'; taking all of the osteosarcomas ascertained (i.e., including those tumours that did not meet the criteria for formal statistical analysis), there were 15 'in the head and neck' and 18 'outside'. Marees et al (2008) also reported that half of their bone cancers in heritable cases were inside the 'irradiation field'. In our study, $42 \%$ of ascertained tumours were in the leg-a finding similar to that reported by Marees et al (2008) who found most of the bone tumours 'outside the irradiation field' were in the legs.

The study by Fletcher et al (2004) reported only one osteosarcoma among the heritable cases. However, they studied only SPTs occurring from the age of 25 years onwards. Most osteosarcomas occur around the mid-teens.

The soft-tissue sarcomas in our study were confined to the heritable group with the exception of one tumour. In our study, and in reports by Marees et al (2008) and Kleinerman et al (2007), leiomyosarcomas were the most common type of soft-tissue sarcoma. Leiomyosarcomas were mainly found in the bladder, uterus and retroperitoneum. The leiomyosarcoma SIRs are very similar for the two sexes. We found 31 leiomyosarcomas in heritable cases, making up $67 \%$ of the soft-tissue sarcomas in our study, in contrast to reports of 35\% and 33\% in Marees et al (2008) and Kleinerman et al (2007), respectively. We found that 24 of the 31 tumours were outside the possible radiotherapy field; which was a similar finding to that reported by Kleinerman et al (2007). Marees et al (2008) reported that most of the soft-tissue sarcomas that developed outside the field of radiation were leiomyosarcomas.

The risk for melanoma of skin in heritable cases was elevated, as previously reported in other studies (Fletcher et al, 2004; Kleinerman et al, 2005; Marees et al, 2008). The risk was higher in males than females, though this difference was not statistically significant. This finding replicates that reported by Marees et al (2008) whose study found a SIR of 109 (95\% CI 52.2-200) for males compared with 18.3 (95\% CI 3.78-53.4) for females, $P=0.01$. The high SIR for males aged $0-14$ years is striking but is based on only one case.

We found twice as many melanomas outside the head and neck region as inside. Marees et al (2008) also reported that most of the melanomas in their study occurred outside the field of radiation. Marees et al (2008) and Kleinerman et al (2005) both reported that they found melanomas in both irradiated and non-irradiated patients.

All of the breast tumours were carcinomas, mainly infiltrating duct carcinomas. One patient developed two separate carcinomas 
Table 7. Site and type for 169 validated subsequent primary tumours in the cohorts of 1927 cases

\begin{tabular}{|c|c|c|c|c|}
\hline Site of SPT & Type of SPT & $\begin{array}{l}\text { No. of heritable } \\
\text { cases }\end{array}$ & $\begin{array}{c}\text { No. of non-Heritable } \\
\text { cases }\end{array}$ & $\begin{array}{l}\text { Total } \\
\text { cases }\end{array}$ \\
\hline Mouth unspecified & Squamous cell carcinoma, NOS & & 1 & 1 \\
\hline Submandibular gland & Adenosquamous carcinoma & & 1 & 1 \\
\hline \multirow[t]{2}{*}{ Nasopharynx unspecified } & Neoplasm, malignant & 1 & & 1 \\
\hline & Carcinosarcoma, NOS & 1 & & 1 \\
\hline Oesophagus unspecified & Adenocarcinoma, NOS & 1 & & 1 \\
\hline Hepatic flexure & Adenocarcinoma, NOS & 1 & & 1 \\
\hline Body of pancreas & Infiltrating duct carcinoma, NOS & 1 & & 1 \\
\hline \multirow[t]{2}{*}{ Nasal cavity } & Spindle cell sarcoma & 1 & & 1 \\
\hline & Leiomyosarcoma, NOS & 1 & & 1 \\
\hline \multirow[t]{2}{*}{ Maxillary sinus } & Fibrosarcoma, NOS & 1 & & 1 \\
\hline & Leiomyosarcoma, NOS & 1 & & 1 \\
\hline \multirow[t]{2}{*}{ Ethmoidal sinus } & Fibrosarcoma, NOS & 1 & & 1 \\
\hline & Leiomyosarcoma, NOS & 1 & & 1 \\
\hline Sphenoidal sinus & Leiomyosarcoma, NOS & 1 & & 1 \\
\hline Accessory sinus unspecified & Leiomyosarcoma, NOS & 1 & & 1 \\
\hline \multirow[t]{3}{*}{ Trachea, bronchus, lung } & Small cell carcinoma, NOS & 3 & & 3 \\
\hline & Carcinoma, NOS & 2 & & 2 \\
\hline & Adenocarcinoma, NOS & 1 & & 1 \\
\hline Mediastinum part unspecified & Precursor cell lymphoblastic lymphoma, NOS & & 1 & 1 \\
\hline \multirow[t]{2}{*}{ Scapula and long bones of upper limb } & Osteosarcoma, NOS & 4 & & 4 \\
\hline & Ewing sarcoma & 1 & & 1 \\
\hline \multirow[t]{3}{*}{ Long bones of lower limb } & Leiomyosarcoma, NOS & & 1 & 1 \\
\hline & Osteosarcoma, NOS & 14 & 2 & 16 \\
\hline & Telangiectatic osteosarcoma & & 1 & 1 \\
\hline \multirow[t]{2}{*}{ Bones of skull and face } & Osteosarcoma, NOS & 13 & & 13 \\
\hline & Chondroblastic osteosarcoma & 2 & & 2 \\
\hline \multirow[t]{4}{*}{ Bone marrow } & Precursor cell lymphoblastic leukaemia, NOS & 2 & & 2 \\
\hline & Acute myeloid leukaemia & 1 & 1 & 2 \\
\hline & Acute monocytic leukaemia & 1 & & 1 \\
\hline & Hairy cell leukaemia & 1 & & 1 \\
\hline Skin of ear and external auricular canal & Melanoma & 1 & & 1 \\
\hline Skin of other and unspecified parts of face & Melanoma & 1 & & 1 \\
\hline Skin of scalp and neck & Melanoma & 3 & & 3 \\
\hline Skin of trunk & Melanoma & 3 & 1 & 4 \\
\hline Skin of upper limb including shoulder & Melanoma & 3 & & 3 \\
\hline Skin of lower limb including hip & Melanoma & 3 & 1 & 4 \\
\hline Malignant neoplasm of skin unspecified & Leiomyosarcoma, NOS & 1 & & 1 \\
\hline Retroperitoneum & Leiomyosarcoma, NOS & 4 & & 4 \\
\hline Specified parts of peritoneum & Leiomyosarcoma, NOS & 1 & & 1 \\
\hline \multirow[t]{5}{*}{ Connective and soft tissue of head face and neck } & Sarcoma, NOS & 1 & & 1 \\
\hline & Liposarcoma, well differentiated & 1 & & 1 \\
\hline & Leiomyosarcoma, NOS & 3 & & 3 \\
\hline & Rhabdomyosarcoma, NOS & 2 & & 2 \\
\hline & Embryonal rhabdomyosarcoma & 2 & & 2 \\
\hline \multicolumn{5}{|l|}{ Connective and soft tissue of lower limb } \\
\hline including hip & Leiomyosarcoma, NOS & 4 & & 4 \\
\hline Connective and soft tissue of thorax & Leiomyosarcoma, NOS & 1 & & 1 \\
\hline \multirow[t]{2}{*}{ Connective and soft tissue of pelvis } & Sarcoma, NOS & 1 & & 1 \\
\hline & Leiomyosarcoma, NOS & 1 & & 1 \\
\hline
\end{tabular}




\section{Table 7. (Continued)}

\begin{tabular}{|c|c|c|c|c|}
\hline Site of SPT & Type of SPT & $\begin{array}{l}\text { No. of heritable } \\
\text { cases }\end{array}$ & $\begin{array}{c}\text { No. of non-Heritable } \\
\text { cases }\end{array}$ & $\begin{array}{l}\text { Total } \\
\text { cases }\end{array}$ \\
\hline Connective and soft tissue of trunk unspecified & Liposarcoma, well differentiated & 1 & & 1 \\
\hline \multirow[t]{3}{*}{ Breast } & Infiltrating duct carcinoma, NOS & 7 & 2 & 9 \\
\hline & Lobular carcinoma, NOS & 1 & & 1 \\
\hline & $\begin{array}{l}\text { Infiltr. duct mixed with other types of } \\
\text { carcinoma }\end{array}$ & 1 & & 1 \\
\hline \multirow[t]{3}{*}{ Uterus } & Leiomyosarcoma, NOS & 5 & & 5 \\
\hline & Endometrioid carcinoma & 1 & & 1 \\
\hline & Endometrial stromal sarcoma & 1 & & 1 \\
\hline \multirow[t]{2}{*}{ Ovary } & Leiomyosarcoma, NOS & 1 & & 1 \\
\hline & Neuroblastoma, NOS & 1 & & 1 \\
\hline Prostate gland & Adenocarcinoma, NOS & & 1 & 1 \\
\hline \multirow[t]{2}{*}{ Testis } & Seminoma, NOS & & 2 & 2 \\
\hline & Mixed germ cell tumour & 1 & & 1 \\
\hline Spermatic cord & Liposarcoma, well differentiated & 1 & & 1 \\
\hline Kidney, NOS & Clear cell adenocarcinoma, NOS & & 1 & 1 \\
\hline \multirow[t]{4}{*}{ Bladder } & Rhabdomyosarcoma, NOS & 1 & & 1 \\
\hline & Transitional cell carcinoma, NOS & 1 & 1 & 2 \\
\hline & Papillary trans. cell carcinoma & 1 & & 1 \\
\hline & Leiomyosarcoma, NOS & 5 & & 5 \\
\hline Orbit & Spindle cell sarcoma & 1 & & 1 \\
\hline \multirow[t]{7}{*}{ Cerebral meninges } & Meningioma, NOS & 4 & & 4 \\
\hline & Meningiomatosis, NOS & & 1 & 1 \\
\hline & Meningioma, malignant & 1 & & 1 \\
\hline & Fibrous meningioma & 2 & & 2 \\
\hline & Hemangioblastic meningioma & 1 & & 1 \\
\hline & Transitional meningioma & & 1 & 1 \\
\hline & Atypical meningioma & 1 & 1 & 2 \\
\hline \multirow[t]{5}{*}{ Frontal lobe } & Peripheral neuroectodermal tumour & 1 & & 1 \\
\hline & Astrocytoma, NOS & & 1 & 1 \\
\hline & Pleomorphic xanthoastrocytoma & 1 & & 1 \\
\hline & Giant cell glioblastoma & 1 & & 1 \\
\hline & Primitive neuroectodermal tumour & 1 & & 1 \\
\hline \multirow[t]{2}{*}{ Temporal lobe } & Rhabdomyosarcoma, NOS & 1 & & 1 \\
\hline & Glioblastoma, NOS & 1 & & 1 \\
\hline \multirow[t]{2}{*}{ Thyroid gland } & Papillary carcinoma, NOS & & 1 & 1 \\
\hline & Papillary carcinoma, follicular variant & 1 & & 1 \\
\hline \multirow[t]{2}{*}{ Head face and neck } & Rhabdomyosarcoma, NOS & 1 & & 1 \\
\hline & Alveolar rhabdomyosarcoma & 1 & & 1 \\
\hline Pelvis & Leiomyosarcoma, NOS & 1 & & 1 \\
\hline Lymph nodes of multiple regions & Hodgkin lymphoma, lymphocyte-rich & & 1 & 1 \\
\hline \multirow[t]{4}{*}{ Unknown primary site } & Neoplasm, malignant & 1 & & 1 \\
\hline & Neuroendocrine carcinoma & 1 & & 1 \\
\hline & Leiomyosarcoma, NOS & 1 & & 1 \\
\hline & Total & 146 & 23 & 169 \\
\hline
\end{tabular}

of the breast, diagnosed 4 years apart. Fletcher et al (2004), Kleinerman et al (2005), and Marees et al (2008) all reported female breast cancers in heritable cases, though the risk estimates quoted were not all significantly raised.
Eight of the SPTs in our study were bladder tumours: five leiomyosarcomas (all diagnosed at age 30 years or later), one rhabdomyosarcoma and two carcinomas; thus individuals with heritable retinoblastoma are at high risk for tumours at this 
site $(\mathrm{SIR}=83.0)$. Our findings are similar to those of Marees et al (2008) who reported a raised risk for bladder cancer, all the bladder cancers being diagnosed at least 30 years after the retinoblastoma diagnosis. The large US series (Kleinerman et al, 2007) reported no leiomyosarcomas at this site. Fletcher et al (2004) reported a SMR of 26.31 based on five cases (four carcinomas; one no histology).

Seven SPTs of the uterus were also found in the heritable cases: five of these were leiomyosarcomas. Tumours of this type were also reported by Kleinerman et al (2007) who suggested that as the uterus is outside the field of radiation, the risk for these tumours may be the result of the RB1 mutation rather than exposure to radiotherapy. Francis et al (2011) examined the occurrence of uterine leiomyosarcomas in detail, and reported that the excess risk was 20 per 10000 women-years for those aged 30-39 years, which increased to 27 for women aged $40+$ years. We report a similar increase in excess risk with age for leiomyosarcoma generally. (Note that risks in our Table 5 are per 100000 women-years.)

The excess risk for lung cancer in heritable cases is well established (Fletcher et al, 2004; Kleinerman et al, 2005; Marees et al, 2008). We found six tumours of the bronchus and lung (though only three of these met our criteria for formal statistical analysis). These tumours were diagnosed between the ages of 43 and 59 years; all six patients died of cancer shortly after the diagnosis of the lung tumour.

In the general population, the risk for lung cancer is related to smoking. Foster et al (2006) carried out a survey of tobacco use in a US cohort of retinoblastoma patients, comparing use by the cohort with that of the general US population, and reported that smoking did not account for the increased risk of lung cancer in heritable cases.

We have demonstrated a particularly high risk for tumours in the head or neck beyond the level seen outside that region; this risk seems likely to be, at least in part, attributable to the effects of radiotherapy. Our results are consistent with there being a multiplicative effect between the SPT risk attributable to the RB1 germline mutation and that for radiotherapy. The finding of eight meningiomas is of particular interest in view of the known association of this tumour with ionising radiation.

An important finding, which has only become clear through the extended follow-up of these patients here and in studies by others, is that heritable retinoblastoma survivors are at particularly high risk of subsequently developing leiomyosarcoma.

Dommering et al (2011) have recently reported an analysis of specific RB1 germline mutations in relation to subsequent tumour risk. Their results, from a population-based cohort, suggest a correlation between genotype and the risk of SPTs. Such findings will be important in the future development of follow-up procedures for retinoblastoma patients.

We plan further studies to explore the effects of treatment, information on which was not available for the present paper. Treatment will vary between years and between hospitals, and of course between countries; this will not affect any of the analyses presented here except, just possibly, those relating to the comparison of SPT rates within and outside the head/neck region. Differences between heritable and non-heritable cases are so extreme that comparisons are unlikely to be much affected by variations in treatment.

\section{ACKNOWLEDGEMENTS}

We are grateful to all colleagues at the Childhood Cancer Research Group, and, in particular, Janette King for help with the manuscript and references. We thank also the National Cancer
Registries, Regional Children's Tumour Registries and the Children's Cancer and Leukaemia Group for providing data to the NRCT. We thank also the clinicians who provided further information on non-ocular tumours. We thank the National Health Service Information Centre, Medical Research Information Service (England and Wales) and the General Register Office (Scotland) for notifications of tumours subsequent to retinoblastoma and the Office for National Statistics and the Welsh Cancer Intelligence and Surveillance Unit for providing us with national cancer data. This work was supported by CHILDREN with CANCER UK, the Department of Health (England and Wales) and the Scottish Government. Oxfordshire Research Ethics Committee (Oxfordshire REC C, Ref 07/Q1606/45) approved the use of the data reported in this study in 2007.

\section{DISCLAIMER}

The above-mentioned organisations had no role in the study design, the collection, analysis and interpretation of data, the writing of the article nor in the decision to submit it for publication.

\section{REFERENCES}

Boice JD, Curtis RE, Kleinerman RA, Flannery JT, Fraumeni JF (1986) Multiple primary cancers in Connecticut, 1935-82. Yale J Biol Med 59(5): 533-545.

Breslow NE, Day NE (1987) Statistical Methods in Cancer Research. Volume 2. The Design and Analysis of Cohort Studies. International Agency for Research on Cancer: Lyon.

Dommering CJ, Marees T, van der Hout AH, Imhof SM, Meijers-Heijboer H, Ringens PJ, van Leeuwen FE, Moll AC (2011) RB1 mutations and second primary malignancies after hereditary retinoblastoma. Fam Cancer 11: 225-233.

Draper GJ, Sanders BM, Kingston JE (1986) Second primary neoplasms in patients with retinoblastoma. Br J Cancer 53(5): 661-671.

Fletcher O, Easton D, Anderson K, Gilham C, Jay M, Peto J (2004) Lifetime risks of common cancers among retinoblastoma survivors. J Natl Cancer Inst 96(5): 357-363.

Foster MC, Kleinerman RA, Abramson DH, Seddon JM, Tarone RE, Tucker MA (2006) Tobacco use in adult long-term survivors of retinoblastoma. Cancer Epidemiol Biomarkers Prev 15(8): 1464-1468.

Francis JH, Kleinerman RA, Seddon JM, Abramson DH (2011) Increased risk of secondary uterine leiomyosarcoma in hereditary retinoblastoma. Gynecol Oncol 124(2): 254-259.

Kleinerman RA, Tucker MA, Abramson DH, Seddon JM, Tarone RE, Fraumeni JF (2007) Risk of soft tissue sarcomas by individual subtype in survivors of hereditary retinoblastoma. J Natl Cancer Inst 99(1): 24-31.

Kleinerman RA, Tucker MA, Tarone RE, Abramson DH, Seddon JM, Stovall M, Li FP, Fraumeni JF (2005) Risk of new cancers after radiotherapy in long-term survivors of retinoblastoma: an extended follow-up. J Clin Oncol 23(10): 2272-2279.

MacCarthy A, Bayne AM, Draper GJ, Eatock EM, Kroll ME, Stiller CA, Vincent TJ, Hawkins MM, Jenkinson HC, Kingston JE, Neale R, Murphy MFG (2009) Non-ocular tumours following retinoblastoma in Great Britain 1951 to 2004. Br J Ophthalmol 93(9): 1159-1162.

Marees T, Moll AC, Imhof SM, de Boer MR, Ringens PJ, van Leeuwen FE (2008) Risk of second malignancies in survivors of retinoblastoma: more than 40 years of follow-up. J Natl Cancer Inst 100(24): 1771-1779.

Online Mendelian Inheritance in Man, OMIM (2012) [MIM Number: 180200]. Johns Hopkins University, Baltimore, MD.

Sasieni PD, Shelton J, Ormiston-Smith N, Thomson CS, Silcocks PB (2011) What is the lifetime risk of developing cancer?: the effect of adjusting for multiple primaries. Br J Cancer 105(3): 460-465.

StataCorp (2009) Stata Statistical Software: Release 11. StataCorp LP: College Station, TX. 
Stiller C (2007) Childhood Cancer in Britain: Incidence, Survival, Mortality. Oxford University Press: Oxford.

Storm HH, Jensen OM, Ewertz M, Lynge E, Olsen JH, Schou G, Østerlind A (1985) Summary: multiple primary cancers in Denmark, 1943-80. Natl Cancer Inst Monogr 68: 411-430.
This work is published under the standard license to publish agreement. After 12 months the work will become freely available and the license terms will switch to a Creative Commons Attribution-NonCommercial-Share Alike 3.0 Unported License. 